\title{
Was tun bei Eisenmangel? Eine neue Publikation hilft
}

Bei oraler Eisentherapie, etwa mit Tabletten, sollten diese nicht täglich eingenommen werden, so Forscher. Auch die richtige Dosis wurde analysiert: Wie sich der Eisenspeicher am besten auffüllen lässt.

In jedem Milliliter Blut sind 0,4$0,5 \mathrm{mg}$ Eisen enthalten. Mit jedem Blutverlust, sei er physiologisch, pathologisch oder iatrogen, geht also Eisen verloren. Die einzige Ursache für Eisenmangel ist das aber nicht. Auch mangelnde Zufuhr (vegetarische Ernährung) oder Resorption, erhöhter Bedarf (während des Wachstums oder in der Schwangerschaft) und körperliche Anstrengung (Eisenverlust im Schweiß) können für ein Eisendefizit (mit)verantwortlich sein.

Eine australisch-niederländischdeutsche Arbeitsgruppe, zu der auch Professor Martina Muckenthaler von der Universität Heidelberg gehört, hat nun das Wissen über den medizinischen Umgang mit Eisenmangel in einer 13-seitigen Publikation zusammengetragen [1].

\begin{abstract}
Was ist heute State-of-the-Art?
„Wir präsentieren in unsererVeröffentlichung Empfehlungen zu State-of-theArt-Diagnostik und Behandlung des Eisenmangels sowie seiner Ursachen", sagt Muckenthaler und weist darauf hin, dass zur Abklärung auch unbedingt dem Verdacht auf eine Zöliakie nachgegangen werden muss. Blutbild und die Bestimmung von Hämoglobin und Ferritin helfen in der Eisenmangeldiagnostik weiter. Die Eisenkonzentration selbst gibt keine Auskunft, ob ein Eisenmangel vorliegt, weil sie auch bei entzündlichen Prozessen erniedrigt sein kann. Für komplexe Fälle gehört zur Diagnostik auch die Aspiration von Knochenmark: Bei absolutem (im Gegensatz zu funktionalem) Eisenmangel ist kein färbbares Eisen mehr im Mark enthalten.
\end{abstract}

Einen erheblichen Teil ihrer Übersicht widmen Muckenthaler und Mitautoren der Behandlung des Eisenmangels. In niedergelassener Praxis dominiert die orale Therapie. Die Einnahme eisenhaltiger Präparate wird dabei einerseits durch gastrointestinale Nebenwirkungen limitiert: Verstopfung, Übelkeit und Durchfälle. Andererseits hat etwa eine hohe Dosierung von 100-200 mg Eisen, aufgeteilt in zwei bis drei Tagesdosen, zur Folge, dass die Konzentration von Hepcidin steigt und die Eisenresorption damit 24 Stunden lang blockiert wird.

\section{Wie sich der Eisenspeicher auffüllen lässt}

Hingegen ist gezeigt worden, dass die Eisenresorption um ein Drittel höher ist, wenn Patienten die Präparate nur alle zwei Tage einnehmen. Zudem kam es bei Patienten mit Eisenmangelanämie unter einer Dosis von $60 \mathrm{mg}$ Eisen zweimal täglich zwar rascher zu einem Anstieg des Hämoglobins. Doch Patienten, die jeden zweiten Tag 120 mg Eisen erhielten, erreichten die gleichen Anstiege, sobald sie die gleiche Gesamtmenge an Eisen aufgenommen hatten - bei geringeren gastrointestinalen Nebeneffekten.

Um sicherzugehen, sind allerdings noch umfangreichere Studien nötig. Muckenthaler und Kollegen halten aber schon einmal fest: „Hohe Eisendosierung und die Aufteilung in zwei bis drei Tagesdosen sind physiologisch ineffizient, die effizienteste Resorption von Eisen ist durch mittlere Dosen im Tageswechsel zu erreichen." Dieses Vorgehen sei bei Patienten mit milden Symptomen und keiner oder mäßig- gradiger Anämie zu empfehlen. Hohe Dosen seien bei schwerem Eisendefizit zu erwägen, weil hier die absolute Resorption steige.

Rasch auffüllen lassen sich die Eisenspeicher bei ausgeprägtem Eisenmangel mit Infusionen. Es gebe mittlerweile exzellente intravenöse Eisentherapien, so Muckenthaler, verabreicht werden sollten sie aber in der Klinik.

\section{Bericht: Robert Bublak}

\section{Literatur}

1. Pasricha SR, Tye-Din J, Muckenthaler MU, Swinkels DW. Iron deficiency. Lancet. 2021 Jan 16;397(10270):233-248. https://doi.org/ 10.1016/S0140-6736(20)32594-0

Hinweis des Verlags. Der Verlag bleibt in Hinblick auf geografische Zuordnungen und Gebietsbezeichnungen in veröffentlichten Karten und Institutsadressen neutral.

J. Gynäkol. Endokrinol. AT 2021 · 31:84 https://doi.org/10.1007/s41974-02100192-w

(c) Springer-Verlag GmbH Austria, ein Teil von Springer Nature 2021 